\title{
The Detection of Impact Damage to the Edges of CFRP Plates Using Extensional Ultrasonic Edge Waves
}

\author{
Jun Yu Harry Chu ${ }^{1}$. Charles R. P. Courtney ${ }^{1}$ (D) \\ Received: 10 February 2021 / Accepted: 22 August 2021 / Published online: 5 October 2021 \\ (c) The Author(s) 2021
}

\begin{abstract}
Extensional edge waves propagate along the edges of plates, with low attenuation in the propagation direction and amplitude decreasing rapidly (within one or two wavelengths) in the direction perpendicular to the plate edge. This makes them an ideal candidate for inspecting the edges of plate-like structures. Here, finite-element models and experiments are used to investigate the propagation and scattering of extensional edge waves in composite plates and application to damage detection is demonstrated. Piezoceramic transducers attached to the edge of a 4-mm-thick carbon-fibre-reinforced polymer (CFRP) plate were used to excite $140-\mathrm{kHz}$ edge waves and damage detection demonstrated using two experimental configurations: pitchcatch and two-transducer pulse-echo. Damage due to edge-on impacts of $5 \mathrm{~J}$ and $10 \mathrm{~J}$ were detected in both configurations. A mass-addition damage model was used to investigate the effect of damage location. Detection was specific to damage within $33 \mathrm{~mm}$ ( 1.5 wavelengths) of the plate edge with propagation unaffected by features beyond that distance. The time of arrival of reflected signals in pulse-echo mode was accurately predicted using the edge-wave group velocity indicating that this configuration can be used for locating damage on edges. The localisation of edge waves means that they can specifically detect damage at the edges of structures, and attenuation measurements indicate that their useful propagation distance is large (5.5 m).
\end{abstract}

Keywords Ultrasonics · Guided waves · Health monitoring · Edge waves

\section{Introduction}

Carbon-fibre-reinforced polymer (CFRP) laminates are increasingly being used in the construction of high performance vehicle panels, particularly fixed-wing aircraft primary structures. However, their potential advantages in terms of weight savings and high yield strength have yet to be fully exploited due to the need for conservative design due to the design risks associated with their susceptibility to impact damage from unanticipated discrete events, which can lead to severe subsurface delamination. In-plane impacts on the edge of the laminate are particularly dangerous as they generate more damage than out-of-plane impact for comparable energies $[1,2]$. One consequence of this is that the potential for lowvelocity impacts to the edges of wing-panel stiffeners due to dropped tools during manufacture or maintenance and the

Charles R. P. Courtney

c.r.p.courtney@bath.ac.uk

1 Department of Mechanical Engineering, University of Bath, Bath BA2 7AY, UK difficulty in inspecting these structures leads to conservative design [2]. Improved non-destructive testing (NDT) and structural health monitoring (SHM) methods for detecting damage at the edge of panels, or other thin structures, is desirable in order to allow weight savings and reduced inspection costs. Ultrasonic edge waves are a guided wave mode that propagates along the edges of thin structures. This behaviour makes them an excellent candidate for inspecting the edges of plates, but they have not been applied to anisotropic layered structures, such as composites. In this work the detection of damage on the edges of CFRP panels using ultrasonic edge waves is modelled numerically and demonstrated experimentally.

The propagation of ultrasonic guided waves (particularly Lamb waves) in plates over large distances and subsequent scattering by damage has been widely investigated $[3,4]$, but has seen limited application near plate edges, where reflection from the edge complicates the interaction with damage. Edge waves are guided waves that propagate parallel to the edge of a structure, with amplitude that decays perpendicular to the edge and so have the potential to address this limita- 
tion in guided wave inspection. At low frequencies (such that the longitudinal wavelength exceeds the thickness) two edge wave modes are present, a symmetrical extensional mode and an antisymmetric flexural mode [5]. Their low-dispersion and low-attenuation behaviour makes both modes attractive for NDT applications. The localisation of the waves to the plate edge allows transmission over substantial distances and simplifies inspection by focusing transmission along the edge, without attempting to inspect the more geometrically complex parts of the inspected structure. For example, provided that the height of a bonded stringer exceeds the penetration depth of the applied edge waves, the geometry, bonding and any filler at the base of the stringer should not affect the transmission, simplifying the inspection of the edges of such components.

Flexural edge waves have been applied to the inspection of structures that taper to a zero-thickness edge. These waves, often referred to as 'wedge waves' were first discovered in the finite element modelling of wedge shaped wave guides in the 1970s [6]. In 2010 an experimental investigation of the interaction of wedge waves with a crack demonstrated that they had potential in NDT and applications for tool edge inspection were proposed [7]. Recent work has experimentally and numerically demonstrated their suitability for inspecting long features with various tapered profiles, with particular application to turbine sealing fins [8].

For flat ended profiles, such as the edges of plates, symmetric extensional edge waves have attracted more attention than flexural waves. These waves are also referred to as 'Rayleigh edge waves', due to their similarity to Rayleigh waves on surfaces. Originally observed in models of rectangular waveguides [9], damage detection using extensional edge waves was demonstrated using laser generated ultrasound in a pitch-catch configuration to detect pitting in steel rail bases in 2012 [10]. Vien et al. numerically modelled the interaction of extensional edge waves in isotropic plates with cracks [11] and developed and experimentally demonstrated the scattering of edge waves by cracks on race-course shaped holes in aluminium plates [12]. Small cracks (less than one fifth of the wavelength) were shown to produce appreciable scattering (into both edge waves and plate modes) with amplitudes varying linearly with crack length. Extensional modes are investigated in this work due to their ease of excitation by piezoelectric plates and high velocity and low dispersion compared to flexural edge waves.

Progress towards application of edge waves in composites has been limited to numerical calculations of their behaviour $[13,14]$. Here generation and detection of edge waves in CFRP composite material is demonstrated experimentally and their use for the detection of on-edge delaminations investigated. The experimental work is supported by finite element modelling directed to: ensuring that the edge waves are correctly identified, elucidating their char- acteristics (principally penetration depth) and clarifying the interaction with the delamination inferred from the experimental results.

Section 2 introduces the general behaviour and analytical solutions for edge waves in isotropic materials, Sect. 3 describes the experimental setup and Sect. 4 describes the finite element model of the system. Experimental and numerical results are presented in Sect. 5 and discussed in Sect. 6.

\section{Background}

Propagating edge waves can have symmetric and antisymmetric (referring to the symmetry of the material motion through the thickness of the plate) modes of various orders in a manner similar to Lamb waves in plates. At low frequencies only the zeroth-order modes are present and, as the ratio of thickness to wavelength tends to zero, they become the thin-plate extensional edge waves and flexural edge waves, respectively. In this work the frequency regime is such that only these lowest-order modes are considered and the excitation is tuned to excite only the extensional/symmetrical mode. This section outlines the theoretical behaviour of extensional edge waves in homogeneous isotropic materials, followed by the expected deviations from this behaviour for composite materials as background to the new numerical modelling and experimental results of this work.

Figure 1 illustrates the extensional edge-wave mode shape in isotropic materials. Figure 1a defines the system of axes. Propagation is in the positive $x$-direction. Figure $1 \mathrm{~b}$ shows the amplitude of displacement oscillation in each direction as the distance from the edge increases; examples of the displacement are shown inset. In each case the wave propagates in the positive $x$-direction (parallel to the plate edge) with elliptical motion in the $x y$-plane that decreases with increasing distance, $y$, from the edge.

In an isotropic material thin enough that the plane stress approximation applies the complex material displacement in the direction of propagation can be shown to be [5]:

$u(x, y)=\frac{i e^{i \xi_{e} k_{\mathrm{p} 1} x}}{k_{\mathrm{p} 1} \gamma \xi_{e}}\left(\xi_{e}^{2} e^{-\gamma k_{\mathrm{p} 1} y}+\gamma \delta A e^{-\delta k_{\mathrm{p} 1} y}\right)$,

and the displacement perpendicular to the surface is:

$v(x, y)=\frac{e^{i \xi_{e} k_{\mathrm{p} 1} x}}{k_{\mathrm{pl}}}\left(e^{-\gamma k_{\mathrm{pl}} y}+A e^{-\delta k_{\mathrm{p} 1} y}\right)$.

In each case the time dependence $e^{-i \omega t}$ is omitted. $c_{\mathrm{pl}}=$ $\sqrt{\frac{E}{\rho\left(1-v^{2}\right)}}$ is the axial-plate-wave velocity for a material of Young's modulus $E$, density $\rho$ and Poisson's ratio $v$ and $k_{\mathrm{pl}}$ is the corresponding wavenumber. The parameters $A, \gamma$ and 


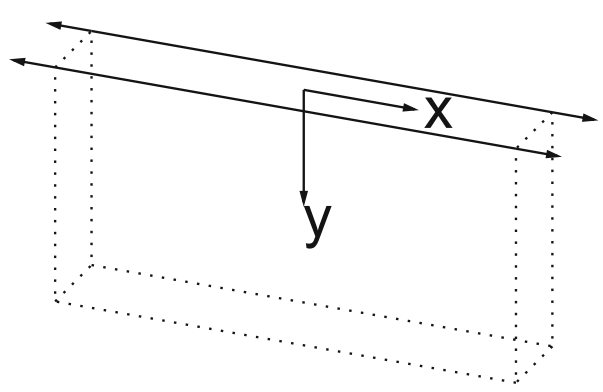

(a)

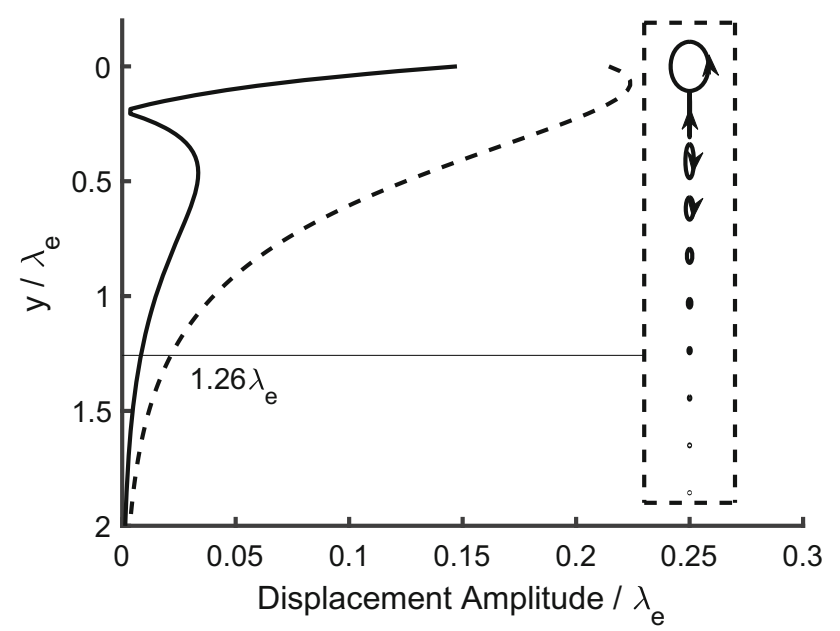

(b)

Fig. 1 a Free edge of thin plate with axes shown. b Amplitude of material displacement as function of depth from surface for edge waves propagating along the edge of a thin isotropic plate with Poisson ratio $v=0.31$. Calculated using Eqs. (1) and (2). Solid line is displacement parallel to $x$-axis, dashed line is parallel to $y$-axis. Displacement paths inset. All distances scaled to the wavelength of the edge wave, $\lambda_{e}$

$\delta$ are given by:

$A=\frac{\xi_{e}^{2} v-\gamma^{2}}{\gamma \delta(1-v)} ; \gamma=\sqrt{\xi_{e}^{2}-1} ; \delta=\sqrt{\xi_{e}^{2}-\frac{2}{1-v}}$,

where $k_{e}$ is the edge-wave wavenumber and the relative wavenumber of the edge wave, $\xi_{e}=\frac{k_{e}}{k_{\mathrm{pl}}}$, is the real solution to

$$
\left(\xi_{e}^{2}+\delta^{2}\right)^{2}-4 \xi_{e}^{2} \delta \gamma=0
$$

The amplitudes of the displacements in Eqs. (1) and (2) are shown in Fig. 1b. The extensional edge wave is nondispersive with a velocity of

$c_{e}=\frac{c_{\mathrm{pl}}}{\xi_{e}}=\frac{1}{\xi_{e}} \sqrt{\frac{E}{\rho\left(1-v^{2}\right)}}$.

It is notable that $\xi_{e}$ depends only on $v$. A straightforward numerical solution of Eq. (4) for Poisson's ratio in the range
$0 \leq v \leq 0.5$ indicates that $\xi_{e}$ varies monotonically from 1.61 to $2.15\left(\xi_{e}=1.86\right.$ for $\left.v=0.31\right)$. So for most practical materials this means that the velocity of the edge wave is considerably slower than the axial plate wave (and hence the low frequency $S_{0}$ Lamb waves that tend to it for thin plates) and faster than the $A_{0}$ Lamb waves that tend to zero velocity at low frequencies.

Equations (1) and (2) indicate that the material displacement forms an ellipse with axes aligned in the $x$ and $y$-directions. The amplitude and aspect ratio vary with depth from the surface according to the parenthetical terms in Eqs. (1) and (2). For motion perpendicular to the plate edge, $v(x, y)$, the amplitude of motion always has the same sign. The behaviour of the motion parallel to the plate edge has a zero crossing near the surface which leads to a change in the direction of material rotation at $y_{0}=$ $\frac{1}{k_{\mathrm{pl}}(\delta-\gamma)} \ln \left(1-\frac{1}{(1-\nu) \xi_{e}^{2}}\right)$. This ranges between $0.17 \lambda_{e}$ and $0.26 \lambda_{e}$ in the region $0 \leq v \leq 0.5$. In Fig. $1 \mathrm{~b}$ this occurs at $y=0.20 \lambda_{e}$, where the zero in the amplitude of the displacement in the $x$-direction can be seen.

Of particular interest for inspection methods is the depth of penetration of the edge wave, i.e. the distance from the edge at which the wave amplitude becomes negligible. From Fig. $1 \mathrm{~b} v(x, y)$ is always greater than $u(x, y)$ so we can take that as the amplitude of interest. For a material with $v=0.31$, $v(x, y)$ drops to $10 \%$ of its peak value at $y=1.26 \lambda_{e}$, which is marked on Fig. $1 b$.

Analytical investigations of edge waves in anisotropic materials are more limited, but their behaviour has been investigated numerically. Ohyoshi considered extensional edge waves in unidirectional fibre-reinforced composites using numerical methods [13]. The general behaviour (even in highly anisotropic materials) was broadly similar to that in isotropic materials, with waves propagating along the edge of the plate with elliptical displacement that decayed with distance from the edge. The main differences observed were that varying the principle axis of the material relative to the edge caused a variation in the edge wave velocity (but it remained non-dispersive) and caused the major axis of the elliptical displacement to become angled to the perpendicular. Cerv et al. used numerical modelling validated with experimental edge wave velocity measurements to investigate the behaviour of edge waves in unidirectional and cross-ply composites [14]. It was confirmed that the waves were non-dispersive, but that velocity depended on the orientation of the fibres relative to the edge.

In summary, the properties that are of particular interest for the development of a structural health monitoring technique are that extensional edge waves are non-dispersive, propagate along the edge of the plate with the largest displacements in the direction perpendicular to the edge and with amplitudes that decay over a distance of the order of a wavelength in 
the direction perpendicular to the edge. The non-dispersive behaviour is important as it allows long distance transmission of pulses without distortion, the displacement direction informs how best to generate and detect edge waves and influences how they will interact with damage and the localisation at the plate edge defines the region that can be inspected.

\section{Experimental Method}

Experiments were performed to determine: the group velocity and attenuation of the edge waves in undamaged CFRP plates, the effect of edge-on impact damage and side-on impact damage on the edge waves, and the effect of damage location on the detected edge wave signals. Methods of using piezoceramic transducers are well known for Lamb waves and have previously been applied successfully to aircraftrelated composite plates [15-19], here edge-mounted piezoceramic transducers were designed to preferentially generate and receive edge waves by tuning their dimensions and frequencies.

Two approaches to damage detection were investigated: pitch-catch and pulse echo. Figure 2 shows the two configurations. In each case the piezoceramic plates were permanently attached to the edge of a CFRP plate to generate and detect edge waves, the damage detection methods differed only in the positioning of the transducers.

In the pitch catch configuration two widely-separated transducers are used, with one transmitting a pulse that propagates along the edge until it is detected by the second transducer. Any damage occurring between the transducers causes scattering of the edge wave and reduces the amplitude of the detected wave.

In the two-transducer pulse-echo mode two transducers are positioned close together with the first sending a pulse. The second transducer detects this pulse almost immediately, followed by later pulses due to reflected waves. In this configuration scattering due to edge damage leads to detectable reflected edge waves. Baseline subtraction is used to identify new features in the received signal. By measuring reflected signals at transducers and calculating the time of flight (TOF), the damage location can be determined. This approach assumes that each echo in the signal was distinct from all others and isolated from interference and coherent noise [20].

In order to demonstrate damage detection, tests were performed using samples impacted on the edge with a drop impactor using energies of $5 \mathrm{~J}, 10 \mathrm{~J}$ and $24 \mathrm{~J}$. To allow investigation of the effect of damage location, two physical models of damage were used: a mass added to the edge of the plate (to simulate on-edge damage) and a clamp across the plate (to simulate side-on damage). Both models were calibrated to the response of real damage created by impact.

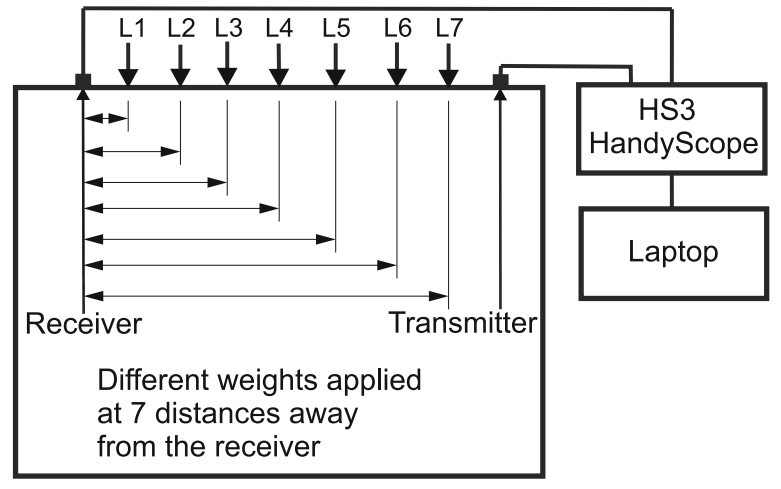

(a)

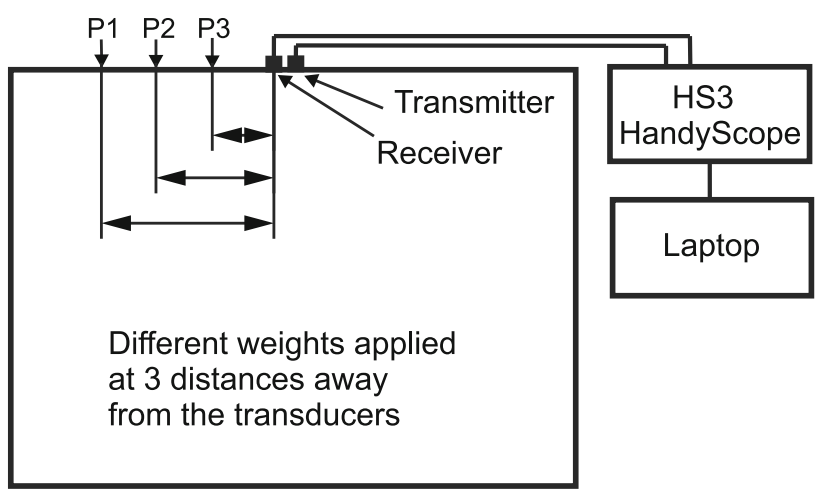

(b)

Fig. 2 Schematic illustration of the experimental setup for edge wave propagation measurements, with locations of simulated damage labelled (L1-7 and P1-3): a pitch-catch, and b two-transducer pulse-echo

\subsection{Materials}

Two sample geometries were fabricated from 16 plies of T800/M21 CFRP in a quasi-isotropic layup: $[(0 /+45 / 90 /$ $\left.-45)_{2}\right]_{s}$. A $600 \times 300 \times 4 \mathrm{~mm}$ plate was used for propagation measurements and measurements using model damage. Three $300 \times 94 \times 4 \mathrm{~mm}$ samples were prepared, and impacted using a drop-weight impact testing machine with a pneumatic anti-bounce mechanism. Figure 2 shows the general configurations for the pitch-catch (Fig. 2a) and two-transducer pulse-echo (Fig. 2b) measurements.

Impact tests were conducted on $300 \times 94 \times 4 \mathrm{~mm}$ samples at three different incident energy levels: $5 \mathrm{~J}, 10 \mathrm{~J}$ and $24 \mathrm{~J}$. The $5 \mathrm{~J}$ and $10 \mathrm{~J}$ on-edge impacts were applied in the plane of the plate at $70 \mathrm{~mm}$ from the transducers, the damage location, sample dimensions and transducer locations are shown in Fig. 3a, b. On the third sample a $24 \mathrm{~J}$ near-edge impact was applied perpendicular to the surface of a sample at a distance of approximately $40 \mathrm{~mm}$ away from the centre point of the plate edge, as shown in Fig. 3c. The edge-on impact energies were selected to give energy/thickness levels $(1.25 \mathrm{~J} / \mathrm{mm}$ and $2.5 \mathrm{~J} / \mathrm{mm}$ ) that are associated of BVID in out-of-plane impacts and comparable with energies for which the effect 


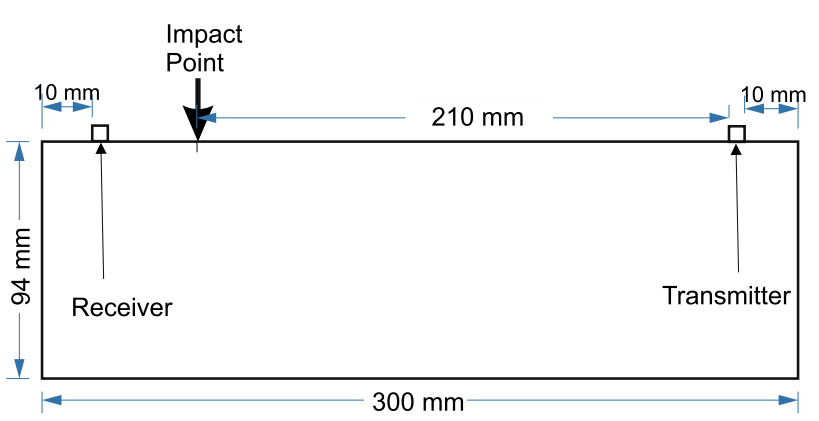

(a)

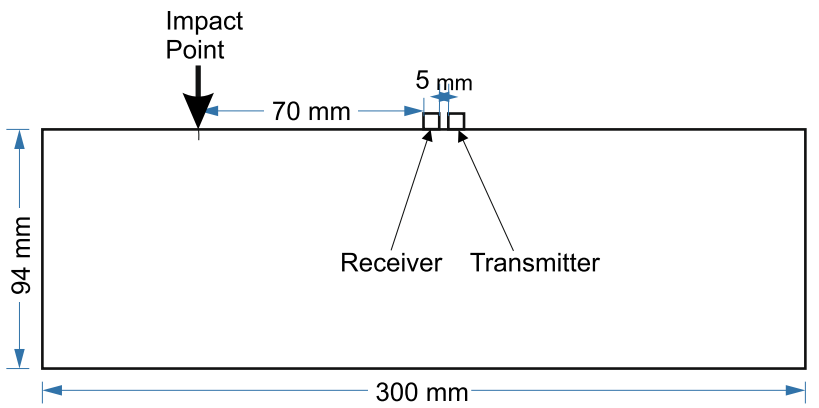

(b)

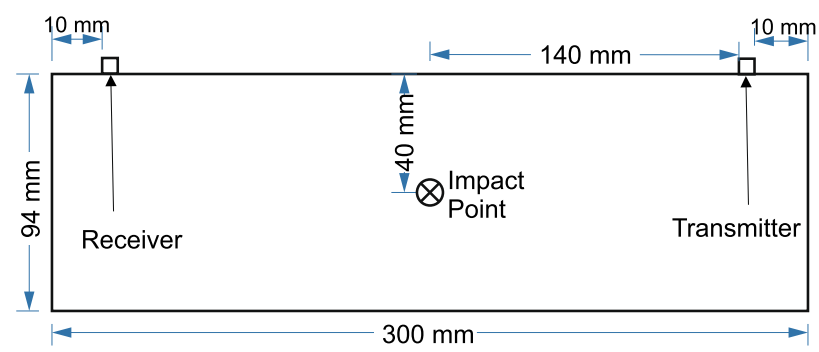

(c)

Fig. 3 Schematic illustration of the location of transducers and impact damage for experimental damage detection tests: a Pitch-catch with edge damage, $\mathbf{b}$ two-transducer pulse-echo with edge damage and $\mathbf{c}$ pitch-catch with side damage

of edge damage has previously been studied $(1.25 \mathrm{~J} / \mathrm{mm}$ in Rhead et al. [2] and 1.7-5.8 J/mm in Ostré et al. [21]) and found to be significant. The out-of-plane energy was selected to induce significant damage to demonstrate the technique's insensitivity to defects away from the edge.

After the impact tests, the damaged area was inspected using a CT scan and an ultrasonic phased-array scan to determine its extent. The phased array (Diagnostic Sonar: 1280.5 -mm-width elements, $5 \mathrm{MHz}$-centre-frequency) was mechanically scanned along the surface of the plate (with coupling gel), while the elements were scanned in the direction perpendicular to the motion, to provide a $\mathrm{C}$ scan of the damaged region.

Each test piece was instrumented using soft-doped PZT plates (NCE51 from Noliac, Kvistgaard, Denmark) of thickness $2 \mathrm{~mm}$ and length $7 \mathrm{~mm}$ and width $4 \mathrm{~mm}$ as shown in Fig.

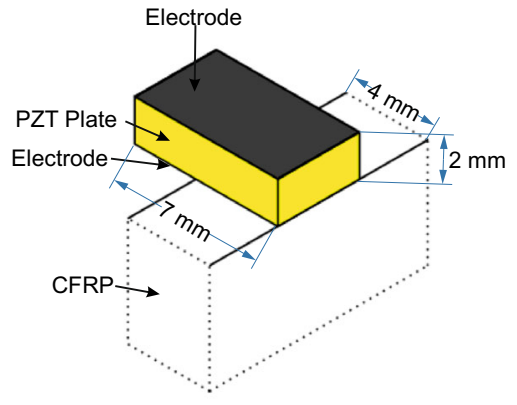

Fig. 4 Dimensions and location of the PZT plates used to generate and receive edge waves

4. Silver electrodes were applied to the top and bottom of the transducers (the $7 \mathrm{~mm}$ by $4 \mathrm{~mm}$ surfaces) and the transducers attached to the plate edge. Placing the transducers perpendicular to the plate edge allowed them to cover the entire width of the composite plate to allow symmetric excitation and access to both sides of the transducer for soldering of connections. The transducers were attached using cyanoacrylate adhesive (Superglue). Two configurations of transducer were used on each plate.

\subsection{Pitch-Catch Configuration}

Figure 2a shows a pair of transducers acting as the transmitter and receiver bonded to the edge of the $600 \times 300 \times 4 \mathrm{~mm}$ plate and separated by $500 \mathrm{~mm}$. Transducers in this configuration were used to determine group velocity dispersion curves, and detect simulated damage at each of seven equally spaced distances (L1-7: $62.5 \mathrm{~mm}, 125 \mathrm{~mm}, 187.5 \mathrm{~mm}, 250 \mathrm{~mm}, 312.5$ $\mathrm{mm}, 375 \mathrm{~mm}$ and $437.5 \mathrm{~mm}$ ). Damage located at different distances from the plate edge was simulated using a G-clamp (clamp diameter: $10 \mathrm{~mm}$ ) to apply a compressive load across the plate surface at a series of points on a line from the centre point (L4 in Fig. 2a) perpendicular to the plate edge.

A similar configuration, with a $280 \mathrm{~mm}$ transducer separation, was used to detect the three different impact damages (on $300 \times 94 \mathrm{~mm}$ samples) and is shown in Fig. 3 .

\subsection{Pulse-Echo Configuration}

For experimental simplicity, a two-transducer method was used, where two piezoceramic plates placed close together were used to make the pulse-echo measurements. This allowed the same instrumentation to be used for both pulseecho and pitch-catch measurements with only the locations of the transducers altered.

Figure $3 \mathrm{~b}$ shows the pulse-echo setup used to detect the response due to the $5 \mathrm{~J}$ and $10 \mathrm{~J}$ edge-on impacts. A pair of transducers acting as the transmitter and receiver are located at the centre of the plate edge (on the $300 \times 94 \mathrm{~mm}$ 
samples) with a 5-mm gap between them and pulse-echo measurements taken before and after the impact damage was introduced.

A similar arrangement (see Fig. 2b) was applied to detecting the location of simulated damage (a $206 \mathrm{~g}$ weight) at each of three equally spaced distances $(62.5 \mathrm{~mm}, 125 \mathrm{~mm}$ and $187.5 \mathrm{~mm}$ ) on the pate edge (of the larger, $600 \times 300 \times 4 \mathrm{~mm}$ plate).

\subsection{Attenuation and Signal-to-Noise Ratio (SNR)}

A scanning laser vibrometer (Polytec, PSV-400) was used to measure the amplitude of the propagating wave at increasing distances from the excitation transducer in order to determine the attenuation. A thin layer of a metallic silver water-based paint was applied to the plate edge in order to improve the consistency of the signal response. Assuming linear attenuation, the amplitude of the decaying wave is:

$A(z)=A(0) e^{-\alpha z}$

where $A(z)$ is the amplitude of the wave at distance $z$, and $\alpha$ is the attenuation coefficient [22]. An exponential curve was fitted, using a non-linear least squares method, to the amplitude as a function of distance to give the attenuation coefficient, $\alpha$.

\subsection{Signal Processing}

The time domain signal was captured at a sample rate of $25 \mathrm{MHz}$, averaged over 100 repetitions and a digital lowpass tenth-order Butterworth filter with a cut-off frequency of $250 \mathrm{kHz}$ applied to reduce unwanted noise in the pulseecho and pitch-catch measurements. The signal envelope was calculated using the Hilbert Transform and used to extract the peak amplitude and pulse arrival time.

For pulse-echo measurements baseline subtraction was used, whereby the residual signal was obtained by subtracting the baseline signal from the received signal. The baseline was recorded when the structure was in a healthy condition, so that the residual signal only includes information about damage.

\section{Finite Element Modelling Method}

The propagation and scattering of edge waves by delaminations was investigated using finite element (FE) models developed in Abaqus/CAE [23]. The numerical analysis was carried out by the Abaqus/Explicit solver.

C3D8R elements (8-node linear brick element) were used to discretise each layer of the composite layup. 16 elements were used through the thickness, one element across each

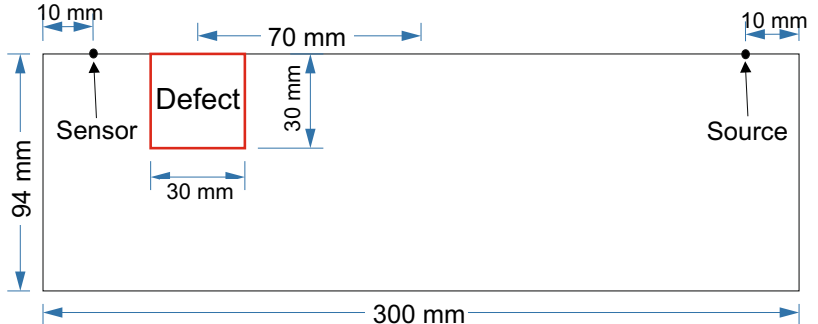

(a)

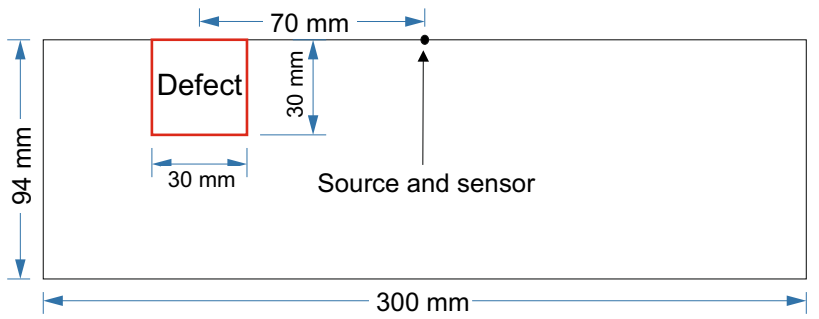

(b)

Fig. 5 Finite element model geometry with damage locations and source and sensor locations marked: a pitch-catch and $\mathbf{b}$ pulse echo

laminae thickness $(0.25 \mathrm{~mm})$, and in the other two dimensions a mesh size of $1 \mathrm{~mm}$ by $1 \mathrm{~mm}$ was selected to be sufficiently small to resolve the smallest wavelength $(11 \mathrm{~mm}$ for the highest frequency in the dispersion curves, $16 \mathrm{~mm}$ for the damaged-plate simulations) and capture the desired highest frequency response. A time increment of $1^{-} \mathrm{s}$ was selected using the Courant-Friedrichs-Levy (CFL) condition [23]. The total number of elements was 450,000 and run times were of the order of 1 hour on a standard desktop computer.

The sensors were represented by single nodes across the plate thickness since they have relatively small size with respect to the distance from the transmitter. The excitation signals generated by the PZT transducer were simulated as displacements perpendicular to the plate edge. The imposed displacement is a 6-cycle sinusoidal Hann-windowed tone burst at $140 \mathrm{kHz}$, which is the same waveform as the electrical signal considered in the experiments (as further discussed in Sect. 5.1). The schematic illustrations for the setup of FE simulations are shown in Fig. 5.

To model multiple delaminations in composite laminates, the plate model was partitioned into two pairs of sub-plates, where a delaminated region (area: $30 \times 30 \mathrm{~mm}^{2}$ ) was defined on the inner contact surfaces of sub-plates (the location is shown in Fig. 5). The inner surfaces at the delamination region were excluded from the connection using a surfaceto-surface tie constraint, in conjunction with the master-slave relationship in Abaqus [23]. This produced discountinuties between the layers of the composite laminated plate. The delaminations were arranged parallel to each other and sym- 


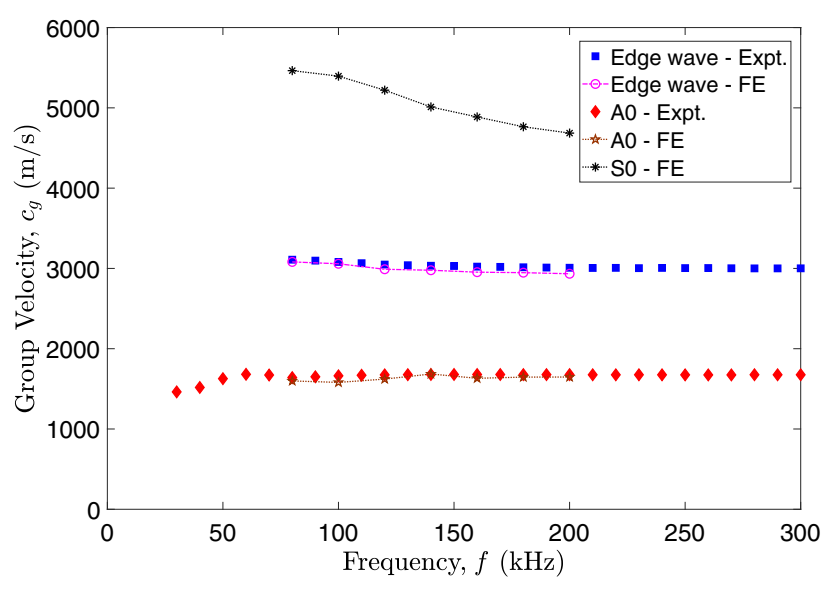

Fig. 6 Comparison of experimental and numerical (Finite Element: Abaqus) group velocities for the 4-mm-thick composite laminated plate described in Sect. 3.1

metrical about the mid-plane of the plate at $1 \mathrm{~mm}$ and $3 \mathrm{~mm}$. The defect location is $70 \mathrm{~mm}$ from the centre of the plate, sharing the same location as the on-edge impact damage.

\section{Results}

The following results start with comparison of the predicted group-velocity dispersion curves with those obtained experimentally to ensure that the correct mode is detected (Sect. 5.1). This is followed by numerical prediction of the shape of the edge wave and its penetration depth in the CFRP sample (Sect. 5.2). Section 5.3 experimentally evaluates the attenuation of the edge wave. Having determined how the edge wave propagates in the sample the ability to detect damage is demonstrated: in Sect. 5.4 established nondestructive methods (ultrasonic C scan and x-ray CT imaging) are used to characterise the induced damage before detection with edge waves is demonstrated in pitch-catch (Sect. 5.5) and pulseecho configurations (Sect. 5.6).

\subsection{Group Velocity Dispersion Curves}

Figure 6 shows the group velocity dispersion curves for edge waves and Lamb waves. The experimental results were obtained from the time-of-flight of the first arrival, using the pitch-catch configuration described in Sect. 3 for edge waves with $15 \mathrm{~mm}$ diameter PZT disk transducers bonded to the plate face to detect Lamb waves. The central frequency of a 6cycle Hann-windowed tone burst signal was increased from $80 \mathrm{kHz}$ to $300 \mathrm{kHz}$ in steps of $10 \mathrm{kHz}$. A smaller number of similar experiments were performed numerically using the finite-element model implemented in Abaqus and described in Sect. 4.

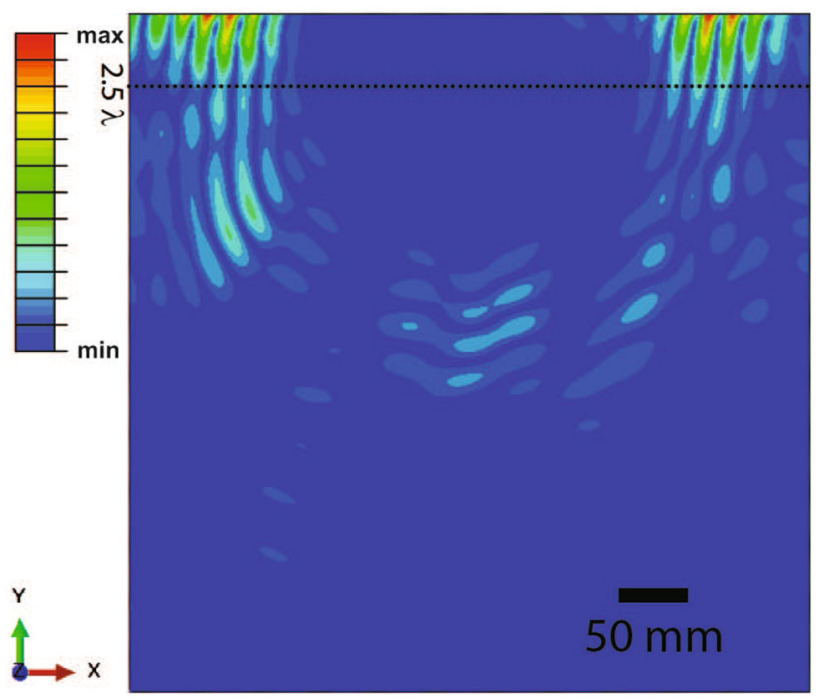

Fig. 7 Displacement amplitude in a $500 \times 500 \mathrm{~mm}^{2}$ quasi-isotropic composite laminated plate $90 \mu$ s after application of a 6-cycle Hannwindowed tone burst at the centre of the top edge. Calculated using the numerical model described in Sect. 4

During the group velocity measurements the edge wave at $140 \mathrm{kHz}$ had the highest amplitude and so was selected for the damage detection application; a 6-cycle Hann-windowed $140-\mathrm{kHz}$ was used in the following sections. This frequency corresponds to a group velocity of $3050 \mathrm{~ms}^{-1}$ and a wavelength of $22 \mathrm{~mm}$ giving ratio of transducer length in the propagation to wavelength of 0.18 .

\subsection{Edge-Wave Mode Shape}

Figure 7 shows the displacement amplitude, obtained from the numerical models described in Sect. 4, $90 \mu$ s after application of the source displacement at the centre of the plate edge. The localised edge waves of interest are apparent on the upper edge. The use of displacements applied perpendicular to the edge as the source also leads to extensional plate waves travelling into the plate and shear horizontal waves at approximately $45^{\circ}$ to the plate edge. The wave field is not symmetrical about the centreline of the plate, in particular the edge waves are both angled in the same direction to the edge. This is due to the absence of reflection symmetry in the lay-up. Maio et al. [24] found similar dependencies of velocity on laminate stacking sequence for Lamb waves in composite laminated plates.

The presence of other wave modes as the edge wave decays makes exact measurement of the penetration depth of the edge wave difficult, but a dashed line at $y=55 \mathrm{~mm}=2.5 \lambda$ in Fig. 7 indicates the approximate penetration depth of the edge wave into the plate. 


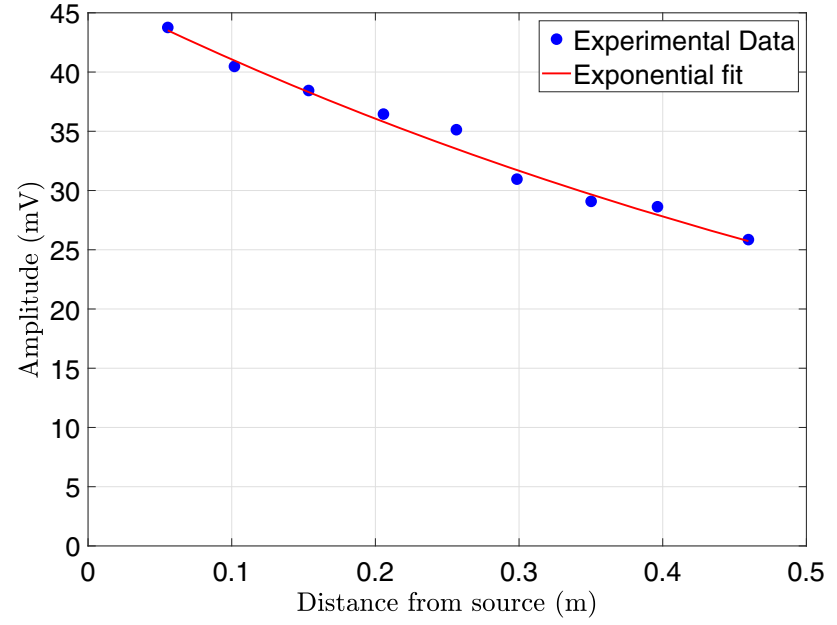

Fig. 8 Laser vibrometer measurements on attenuation over distance along the plate edge

\subsection{Attenuation}

Figure 8 shows the pulse amplitude as a function of distance with Eq. 6 fitted using a non-linear least squares method. The unattenuated amplitude $A_{0}$ with $95 \%$ confidence bounds is $0.040 \pm 0.001$ and the attenuation coefficient $\alpha$ is $1.3 \pm 0.1$.

The measured noise of the processed signal received at 0.5 $\mathrm{m}$ from the source was $0.0004 \%$ of the signal amplitude (signal to noise ratio $108 \mathrm{~dB}$ ). Assuming that the noise remains constant with distance and the signal attenuation continues at the above rate, useful information can be obtained using transducers with large separations. The noise will be $1 \%$ of the signal (signal to noise ratio: $40 \mathrm{~dB}$ ) at $5.5 \mathrm{~m}$ transducer separation for pitch-catch measurements.

\subsection{Characterisation of Damage}

Figure 9 shows the damage introduced to the $300 \times 94 \times$ $4 \mathrm{~mm}$ plates, after baseline measurements had been taken at $140 \mathrm{kHz}$. The on-edge impacts (at 5-J and 10-J) introduced visible dents on the impacted surface as seen in Fig. 9a, b. The near-edge CT image in Fig. 9c shows a 2D slice near (at a depth of $1.7 \mathrm{~mm}$ ) the edge of the plate. Delaminations can be observed for both 5-J and 10-J impacts. For the 5-J impact, delaminations extend approximately $17 \mathrm{~mm}$ along the edge and for the 10-J impact, they extend $38 \mathrm{~mm}$. These lengths correspond to $0.75 \lambda$ and $1.7 \lambda$ respectively at $140 \mathrm{kHz}$. The delaminations occurred at symmetrical positions about the mid-thickness of the specimen at depths of $1 \mathrm{~mm}$ and $3 \mathrm{~mm}$ from the top surface.

The $24 \mathrm{~J}$ side-on impact perpendicular to the plate surface produced damage that showed little change on the surface, but considerable internal disruption. Figure $9 \mathrm{c}$ shows an ultra-

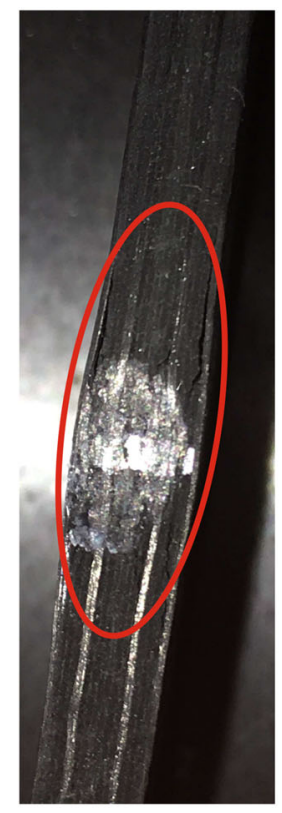

(a)

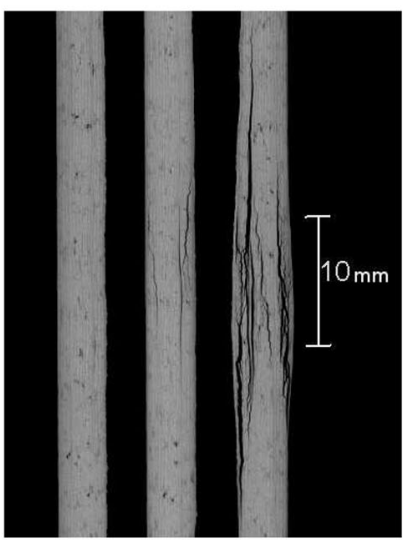

(c)

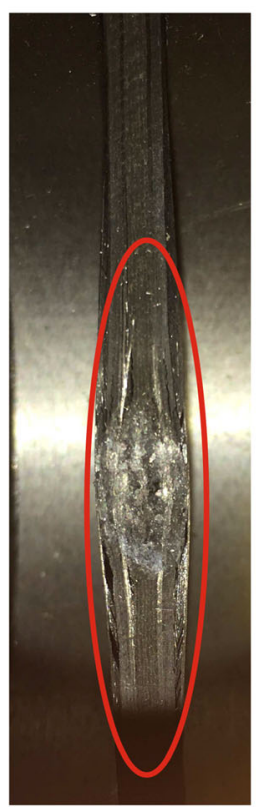

(b)

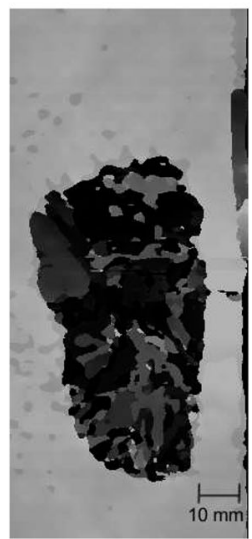

(d)
Fig. 9 Localised views of 4-mm-thick laminate illustrating damage from impact (not-to-scale): a on-edge impact damage $5 \mathrm{~J}$ and $\mathbf{b} 10 \mathrm{~J}$, c out-of-plane view of CT scan image: No damage (left); On-edge impact damage $5 \mathrm{~J}$ (centre) and $10 \mathrm{~J}$ (right). d In-plane view of phased-array image: Near-edge side-on impact damage $24 \mathrm{~J}$

sonic phased array image the resulting damaged region. The extent of the delamination is shown: the nearest boundary is approximately $10 \mathrm{~mm}$ from the plate edge and the dimensions of the damage are $80 \mathrm{~mm}$ parallel to the edge and up to $40 \mathrm{~mm}$ perpendicular.

\subsection{Pitch-Catch Method}

The effect of damage on the amplitude of transmitted edge waves in the pitch-catch configuration described in Sect. 3.2 was assessed by comparing the envelope of the received signal of the baseline (before damage) with that after the impact damage was introduced. In each case the signal was a 6-cycle 

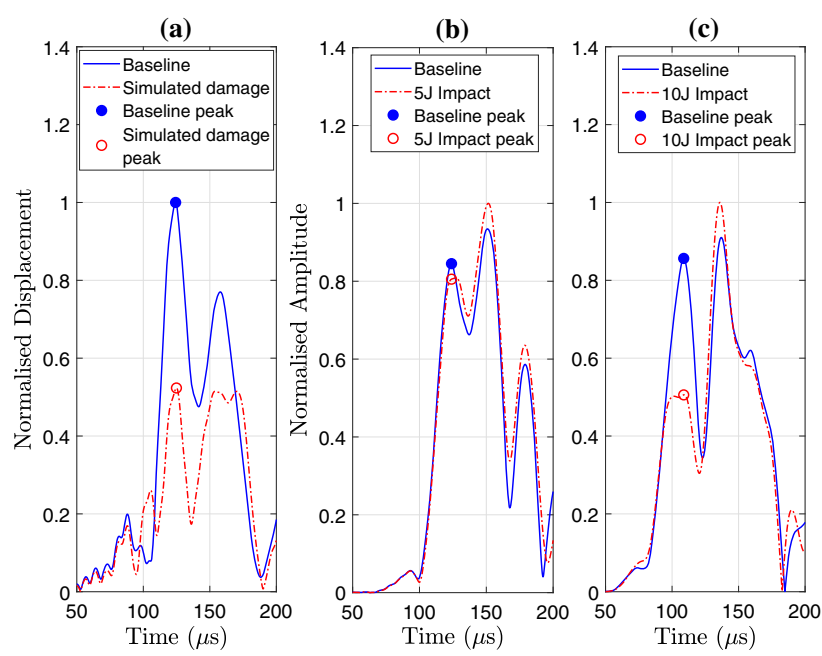

Fig. 10 Effect of on-edge impact damage on pitch-catch responses; Comparison of the normalised envelope for the composite specimen (size: $300 \times 94 \times 4 \mathrm{~mm}$ ) obtained from a FE model with multiple delaminations; and from the experiments for the impacted plates at $\mathbf{b} 5 \mathrm{~J}$, and c $10 \mathrm{~J}$ incident energy levels, located at approximately $70 \mathrm{~mm}$ from the transducers

$140-\mathrm{kHz}$ toneburst. Figure 10 compares the envelope of the first arrival of the pitch-catch signal responses in three scenarios; the FE simulation and experimental values for the two edge-impacted plates. In each case the signals are scaled to give a peak value of the undamaged signal equal to one. The FE model results shown in Fig. 10a indicate a 48\% decrease in the out-of-plane displacement of the local maximum of the first arrival at $125 \mu \mathrm{s}$, and then a second local maximum at $158 \mu$ s reduced by $33 \%$. Figure $10 \mathrm{~b}, \mathrm{c}$ display the amplitude of the envelopes from the signal responses for $5 \mathrm{~J}$ and $10 \mathrm{~J}$ on-edge impacts and their respective baselines. The changes in first-arrival peak amplitude are $4 \%$ and $41 \%$ respectively.

There is a small peak at $90 \mu$ s corresponding to the $S_{0}$ mode reflected at the back face and on to the receiver. Though small in this configuration, the $S_{0}$ is sufficiently large in the pulse-echo configuration to necessitate the use of baseline subtraction for those measurements. Another peak appears $30 \mu \mathrm{s}$ after the direct edge wave. In the model result (Fig. 10a) this peak is reduced by the defect in a similar manner to the direct pulse, but it is largely unaffected by the impact damage in the experimental results. This time of flight corresponds to the edge wave initially travelling away from the receiver before reflecting from the plate edge and equivalently the pulse that passes the receiver before returning from the other edge. Beyond this second arrival the wave field becomes increasingly complex due to multiple reflections from the ends of the plates and mode conversions at reflection.

To investigate the effect of defect location, a damage proxy was required. After preliminary tests, a $206 \mathrm{~g}$ weight was selected as giving results most similar to those seen for the 10

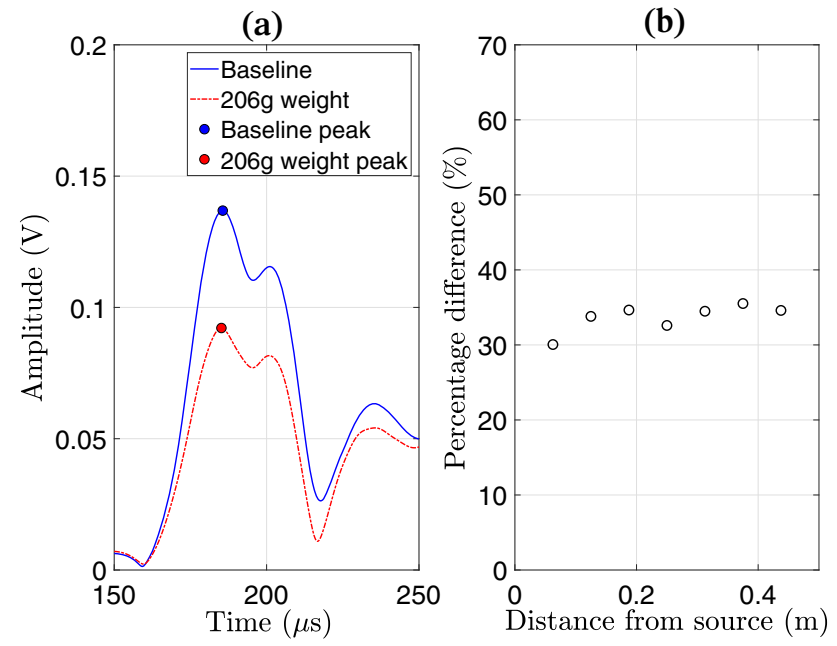

Fig. 11 Effect of adding masses to the plate edge on pitch-catch signals. a Comparison of the envelope from the baseline signal and signal response with $206 \mathrm{~g}$ weight, loaded on the plate edge at $250 \mathrm{~mm}$. b The percentage difference of the local maxima extracted from the envelopes with the same mass, loaded on the plate edge at various distances from the source

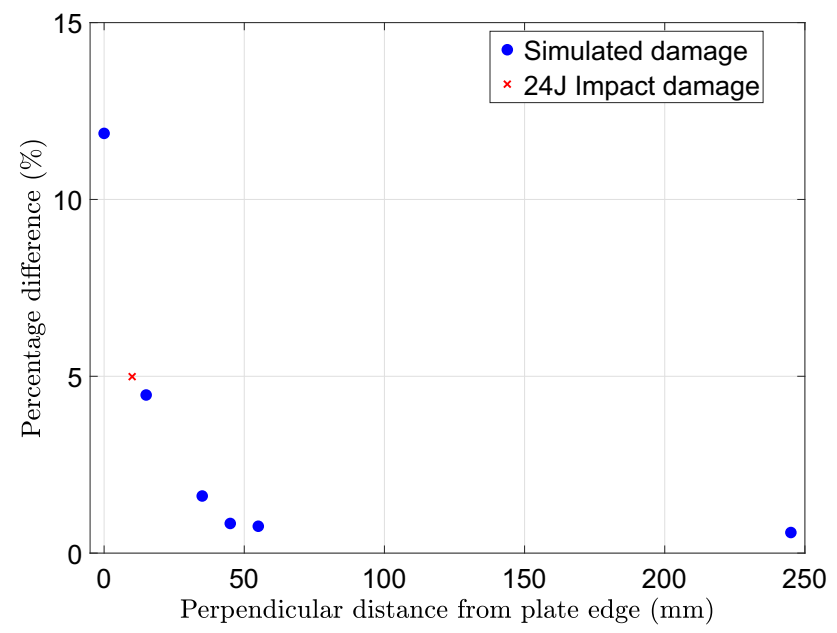

Fig. 12 Effect of applying load and 24-J side-on near-edge impact damage on plate surface on pitch-catch signals; The percentage difference of the local maxima extracted from the envelopes with a G-clamp, applied to plate surface with varying distances from the edge

J impact damage. Figure 11a presents two different envelopes from the signal responses, with and without the $206 \mathrm{~g}$ weight. A decrease of $33.5 \%$ in the peak amplitude was observed relative to the baseline. Repeating this measurement (replacing the same mass at the same point each time) resulted in a distribution of peak amplitudes with a standard deviation of $0.6 \%$. As in the modelled results the second edge-wave peak is reduced by the presence of the mass. The effect of varying the weight location is shown in Fig. 11b: the percentage change in peak amplitude due to the weight at different locations varied between 31 and $37 \%$. 


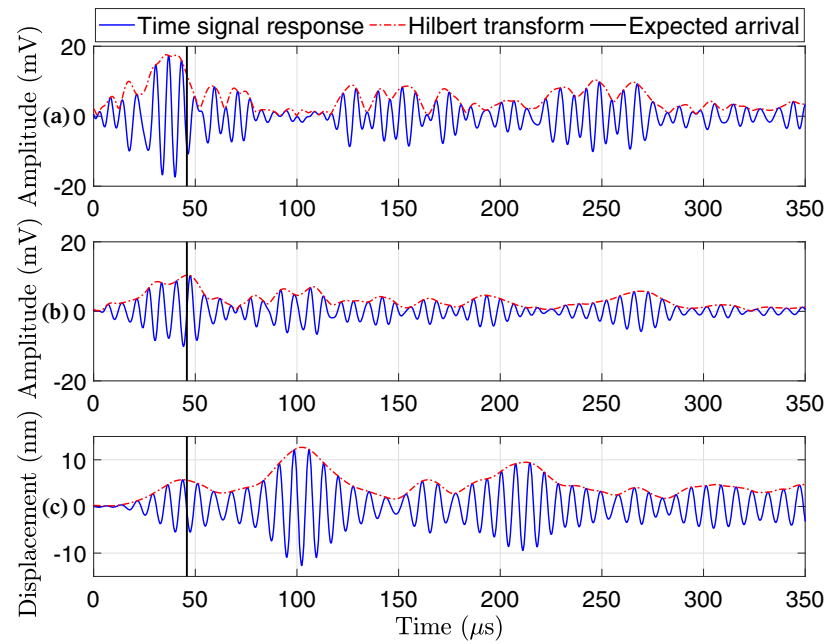

Fig. 13 Pulse-echo responses after baseline subtraction for impact damaged plate edge at $70 \mathrm{~mm}$ from the transducers: a $5 \mathrm{~J}$ impact, b $10 \mathrm{~J}$ impact, c FE model with multiple delaminations

Similarly a proxy was needed to investigate sensitivity to damage with increasing distance from the plate edge. Figure 12 shows the effect on the pitch-catch signal of a clamp across the plate at increasing distances from the edge. Where the clamp is located at the edge it reduces the transmitted signal by $12 \%$. This effect decreases to $2 \%$ as the clamp approaches $35 \mathrm{~mm}$ from the edge, which is approximately one-and-half wavelengths $(33 \mathrm{~mm})$ from the edge. At distances greater than $45 \mathrm{~mm}(2 \lambda)$ the percentage change settles at $0.6 \%$. At $10 \mathrm{~mm}$ from the edge the resulting change in amplitude is similar to the $5 \%$ change seen in the the $24 \mathrm{~J}$ near-edge impact damage, which extends from $10 \mathrm{~mm}$ from the plate edge.

\subsection{Pulse-Echo Experimental Results}

In the pulse echo method of detecting damage, baseline subtraction was used to produce a residual signal that measures the waves scattered by damage in the sample back to the transducer location (source and receiver were located close to each other).

Figure 13 demonstrates the detection of the impact damage using the pulse-echo response. After the damage has been introduced, a peak occurs near the expected arrival time for a defect $70 \mathrm{~mm}$ from the transducers $(45 \mu \mathrm{s}$, marked with a vertical black line) in both Fig. 13a, b. For the $5 \mathrm{~J}$ impact the peak amplitude is at $41.5 \mu \mathrm{s}$, corresponding to a reflector at a distance $63 \mathrm{~mm}$ to the transducer. For the $10 \mathrm{~J}$ impact the maximum signal occurs at $45 \mu \mathrm{s}$ with another peak at $31 \mu \mathrm{s}$, which corresponds to a distance from of $47 \mathrm{~mm}$. Due to the broadly one-dimensional propagation, there are also changes in the signal at times after the arrival of the reflected signal, as all reflections from the end of the plate also pass through the damaged region.
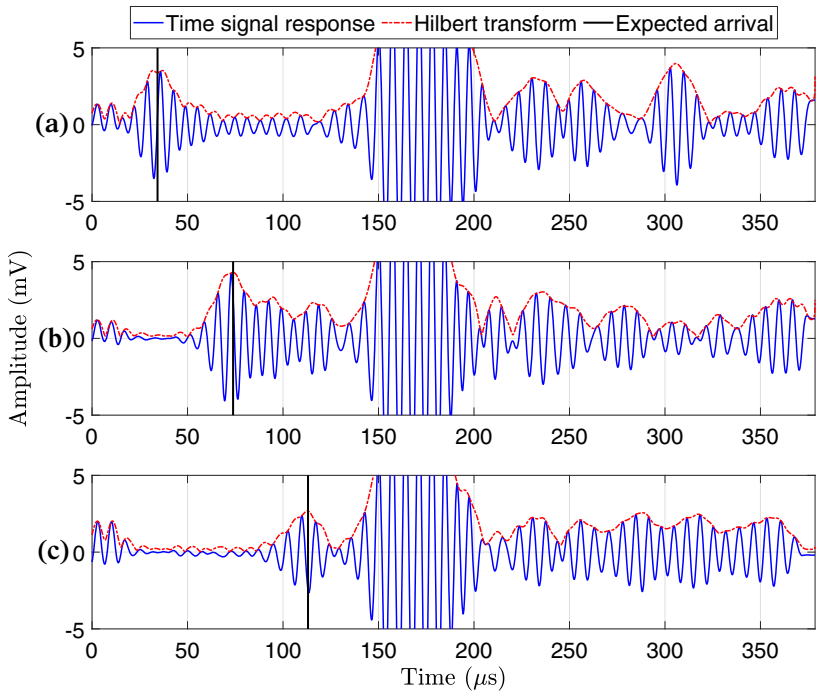

Fig. 14 Pulse-echo responses after baseline subtraction with $206 \mathrm{~g}$ added at various distances from the transducers: a $62.5 \mathrm{~mm}$, b 125 $\mathrm{mm}$; and c) $187.5 \mathrm{~mm}$

The simulation results in Fig. 13c also confirms that the local maximum aligned with the arrival time of the reflected pulse from the discontinuities.

Figure 14 presents the residual signals left after baseline subtraction using the 206-g mass damage model. The vertical black solid lines represent the expected TOF from the mass location, calculated as the time taken to cover the known distance to the simulated damage and back at the group velocity of the edge wave $(3050 \mathrm{~m} / \mathrm{s}$ at $140 \mathrm{kHz})$. Figure $14 \mathrm{a}-\mathrm{c}$ show that the arrival time of the wave packet varies with the distance of the weight as predicted by the group velocity. In each case a large residual signal occurs just after 150 $\mu s$ (independent of defect location). This corresponds to the expected arrival time of the reflection of the plate edge behind the defect $(163 \mu s)$. This reflected pulse passes through the damaged region twice leading to a substantial decrease in amplitude (of the order of that observed in pitch-catch measurements) which appears as a large pulse in the residual signal.

\section{Discussion}

Using transducers in a pitch-catch configuration, it was seen that edge-on impacts cause an appreciable reduction in amplitude in a signal received after passing through the damage region, indicating that plate edge damage can be detected by this method. For a $10 \mathrm{~J}$ impact this decrease amounted to $41 \%$ of the amplitude of the first arrival pulse in the undamaged case. This reflects the significant delaminations and matrix cracking observed in Fig. 9. For the $5 \mathrm{~J}$ case, with less extensive damage, the reduction in amplitude was $4 \%$, which is 
less obvious in the plotted data, but exceeds the variation seen in repeated measurements of $0.6 \%$. Using an edge mounted mass, selected to give a similar response to the $10 \mathrm{~J}$ damage it was shown that the change in the signal was approximately the same regardless of the position of the simulated damage between the transducers. A second-arrival edge wave signal is received shortly after the first due to reflections from the plate edge. In the model and simulated damage results this behaves as might be expected, with a reduction in amplitude when the simulated damage is added. For the plate with 10J-impact damage the second arrival seems unaffected by the damage. This behaviour is unexpected and may relate to the symmetrical location of the transducers leading to a complex interaction of two or more paths.

Pulse-echo measurements demonstrated that edge waves successfully detected impact damage at both $5 \mathrm{~J}$ and $10 \mathrm{~J}$. The arrival time of the first reflected pulse in each damage case $(41.5 \mu \mathrm{s}$ and $31 \mu \mathrm{s}$, respectively) can be converted to a distance using the group velocity and gives distances of 63 $\mathrm{mm}$ and $47 \mathrm{~mm}$. These values agree well with the separation of the transducers and the nearest edge of the damage (61 $\mathrm{mm}$ and $51 \mathrm{~mm}$ respectively) obtained from the images in Fig. 9a-c. The prediction of the damage location was further explored using simulated damage (added mass) and the time of arrival of the reflected waves accurately can be predicted using the time of flight, at a range of positions.

In order to demonstrate the localisation of the waves at the plate edge and the specificity of the technique to damage at the edge, simulated damage consisting of a clamp tightened across the plate was used. $24 \mathrm{~J}$ impact damage centred on 40 $\mathrm{mm}$ from the edge resulted in a delamination extending to 10 $\mathrm{mm}$ from the edge was used to indicate that the effect of the clamp is comparable to real damage. As the clamp head was moved away from the edge, the change in the signal reduced to reach a level of approximately $0.5 \%$ at approximately 45 $\mathrm{mm}(\approx 2 \lambda)$ and did not decrease further. This is of the order that might be expected from solutions to Eqs. (2) and (4), which analytically determine the oscillation of the edge wave perpendicular to the plate in isotropic materials and indicate that the amplitude drops to $1 \%$ of its peak (on-edge) value at 2.2 $\lambda$. The experimental results are also compatible with the penetration depth obtained from the FE model of the quasiisotropic plate (see Fig. 7) which is approximately $2.5 \lambda$.

The finite-element model uses a line source resulting in significant generation of Lamb waves that travel into the plate, as seen in Fig. 7. The experimental method uses transducers of finite width $(4 \mathrm{~mm})$ in the edge-wave propagation direction and the frequency of operation $(140 \mathrm{kHz})$ is selected to maximise the edge-wave amplitude. This provides sufficient specificity in generating edge waves (rather than Lamb waves) to demonstrate edge damage detection in the pitchcatch configuration. However, the generation of some Lamb Waves which are reflected at the opposing edge of the panel to the transducers, requires that baseline subtraction is used for the pulse-echo measurements. There is potential for baselinefree inspection if greater mode-specificity can be achieved. Wedges and inter-digital transducers are methods that merit investigation to achieve this.

The one-dimensional propagation of the waves along the edge allows large transmission distances, with only the attenuation of the waves reducing their amplitude, in contrast to the reduction of amplitude due to two dimensional spreading in guided waves. The attenuation coefficient of $1.3 \pm 0.1 \mathrm{sug}$ gests that transducer separations of up to $5.5 \mathrm{~m}$ are possible.

\section{Conclusions}

The experimental and numerical results presented provide demonstrate the viability of damage detection with extensional edge waves in composite components and provide an investigation of the propagation behaviours relevant to edgedamage detection.

The attenuation of the edge wave was measured experimentally and showed that edge waves are suitable for NDT and SHM applications over long distances (up to $5.5 \mathrm{~m}$ ). Both pitch-catch and two-transducer pulse-echo configurations were shown to be able to detect $5 \mathrm{~J}$ and $10 \mathrm{~J}$ on-edge impact damage, with the pulse-echo response demonstrating the ability to locate the damage. Pitch-catch and pulse-echo detection methods were demonstrated over a range of damage locations using added mass as a damage model. The edgewave detection method was specific to damage near (within $33 \mathrm{~mm}$ ) to the edge and detectability did not vary with location along the edge.

The localised inspection of edges using permanantly attached sensors allows regions to be monitored that are difficult to reach with Lamb waves and has the potential to allow targeted monitoring of important parts of structures while avoiding wave propagation in areas that are of less interest, but complicate the signals significantly. The use of extensional edge waves to inspect anisotropic materials significantly broadens the range of materials and geometries that can be inspected with guided edge waves.

Acknowledgements The authors gratefully acknowledge funding from the EPSRC and technical support from Tom Bertenshaw at GKN as part of the Rapid Inspection of Complex Geometries Using Edge-Guided Ultrasonic Waves project (Grant No. EP/M027724/1).

Open Access This article is licensed under a Creative Commons Attribution 4.0 International License, which permits use, sharing, adaptation, distribution and reproduction in any medium or format, as long as you give appropriate credit to the original author(s) and the source, provide a link to the Creative Commons licence, and indicate if changes were made. The images or other third party material in this article are included in the article's Creative Commons licence, unless indicated otherwise in a credit line to the material. If material 
is not included in the article's Creative Commons licence and your intended use is not permitted by statutory regulation or exceeds the permitted use, you will need to obtain permission directly from the copyright holder. To view a copy of this licence, visit http://creativecomm ons.org/licenses/by/4.0/.

\section{References}

1. Malhotra, A., Guild, F.J., Pavier, M.J.: Edge impact to composite laminates: experiments and simulations. J. Mater. Sci. 43(20), 6661 (2008)

2. Rhead, A.T., Marchant, D., Butler, R.: Compressive strength of composite laminates following free edge impact. Compos. Part A 41, 1056-1065 (2010)

3. Cawley, P., Alleyne, D.: The use of Lamb waves for the long range inspection of large structures. Ultrasonics 34(2), 287 (1996)

4. Wilcox, P., Lowe, M., Cawley, P.: The effect of dispersion on longrange inspection using ultrasonic guided waves. NDT\&E Int. 34(1), $1(2001)$

5. Lawrie, J.B., Kaplunov, J.: Edge waves and resonance on elastic structures: an overview. Math. Mech. Solids 17(1), 4 (2012)

6. Lagasse, P.E.: Analysis of a disperstonfree guide for elastic waves. Electron. Lett. 8(15), 372 (1972). https://doi.org/10.1049/ el:19720271

7. Liu, I.H., Yang, C.H.: in An investigation on wedge waves and the interaction with a defect using aquantitative laser ultrasound visualization system. In: Proceedings-IEEE Ultrasonics Symposium (2010), pp. 817-820

8. Corcoran, J., Leinov, E., Jeketo, A., Lowe, M.J.S.: A guided wave inspection technique for wedge features. IEEE Trans. Ultrasonics Ferroelectr. Freq. Control 67(5), 997 (2020)

9. Lagasse, P.E.: Higher-order finite-element analysis of topographic guides supporting elastic surface-waves. J. Acoust. Soc. Am. 53(4), $1116(1973)$

10. Cerniglia, D., Pantano, A., Vento, M.: Guided wave propagation in a plate edge and application to NDI of rail base. J. Nondestruct. Eval. 31(3), 245 (2012)

11. Vien, B.S., Nadarajah, N., Francis Rose, L.R., Chiu, W.K.: Scattering of the symmetrical edge-guided wave by a small edge crack in an isotropic plate. Int. Workshop Struct. Health Monit. (IWSHM) 2, 1965-1972 (2015)

12. Vien, B.S., Francis Rose, L.R., Chiu, W.K.: Experimental and computational studies on the scattering of an edge-guidedwave by a hidden crack on a racecourse shaped hole. Materials 10, 7 (2017)
13. Ohyoshi, T.: The propagation of Rayleigh waves along an obliquely cut surface in a directional fiber-reinforced composite. Compos. Sci. Technol. 60(12-13), 2191 (2000)

14. Cerv, J., Kroupa, T., Trnka, J.: Influence of principal material directions of thin orthotropic structures on Rayleigh-edge wave velocity. Compos. Struct. 92(2), 568 (2010)

15. Giurgiutiu, V.: Structural health monitoring with piezoelectric wafer active sensors. Structural Health Monitoring with Piezoelectric Wafer Active Sensors (Elsevier Science, 2008)

16. Ihn, J., Chang, F.: Pitch-catch active sensing methods in structural health monitoring for aircraft structures. Struct. Health Monit. 7(1), 5 (2008)

17. Giurgiutiu, V., Zagrai, A., Jing Bao, J.: Piezoelectric wafer embedded active sensors for aging aircraft structural health monitoring. Struct. Health Monit. 1(1), 41 (2002)

18. Giurgiutiu, V.: Tuned lamb wave excitation and detection with piezoelectric wafer active sensors for structural health monitoring. J. Intell. Mater. Syst. Struct. 16, 291 (2005)

19. Gresil, M., Giurgiutiu, V.: Guided wave propagation in composite laminates using piezoelectric wafer active sensors. Proc. SPIE 8695, 971 (2013)

20. Cawley, P., Cegla, F., Galvagni, A.: Guided waves for NDT and permanently-installed monitoring. INSIGHT 54(11), 594 (2012)

21. Ostré, B., Bouvet, C., Minot, C., Aboissière, J.: Experimental analysis of CFRP laminates subjected to compression after edge impact. Compos. Struct. 152, 767 (2016). https://doi.org/10.1016/ j.compstruct.2016.05.068

22. Lempriere, B.M.: 3-wave propagation concepts. In: Ultrasound and Elastic Waves: Frequently Asked Questions, pp. 25-44. Academic Press, Washington (2003)

23. ABAQUS, Abaqus/CAE User's Guide. Abaqus/CAE User's Guide. SIMULA, 2016th edn. (2016)

24. Maio, L., Memmolo, V., Ricci, F., Boffa, N.D., Monaco, E.: Investigation on fundamental modes of guided waves propagating in symmetric and nonsymmetric composite laminates. Proc. Inst. Mech. Eng. Part C 231(16), 2988 (2017)

Publisher's Note Springer Nature remains neutral with regard to jurisdictional claims in published maps and institutional affiliations. 